\title{
SELF-INJECTIVE RINGS
}

\author{
E.T. Wong and R.E. Johnson*
}

(Communicated by G.D. Findlay and J. Lambek, June 10, 1959)

Historically, the first example of a ring of quotients was the quotient field of an integral domain. Later on, conditions were found under which a noncommutative integral domain has a quotient division ring. More recently, R.E. Johnson [4], Y. Utumi [5], and G.D. Findlay and J. Lambek [3] have discussed the existence and structure of a maximal ring of quotients of any ring.

The present paper uses the methods of Findlay and Lambek to recast the results of Johnson on the quotient ring of a ring with zero singular ideal. It is also shown that such a ring has a unique left-right maximal ring of quotients.

For simplicity, each ring $R$ considered is assumed to have a multiplicative identity element and each $\mathrm{R}$-module is assumed to be unitary. If $C$ and $M$ are right $R$-modules, then $M$ is called an essential extension of $C$ if $M \supset C$ and $x R \cap C \neq 0$ for every nonzero $x$ in $M$. If $M \supset C$, then $M$ is called an injective extension of $C$ if every homomorphism of any module $A$ into $M$ can be extended to a homomorphism of $B$ into $M$ where $B$ is any module containing $A$. It is known [1] that every $R$-module $C$ has a unique (up to isomorphism over C) maximal essential extension $E$ that at the same time is the unique minimal injective extension of $C$.

DEFINITION 1. The module $M$ is called a rational extension of $C$ if: $\quad$ i. $\quad M \supset C$ as right $R$-modules, and ii. If $M \supset B \supset C$ and $f \in \operatorname{Hom}_{R}(B, M)$, with $f(C)=0$ then $f=0$. * The authors wish to thank J. Lambek for his criticisms and
suggestions.

Can. Math. Bull. vol, 2, no. 3, Sept. 1959 
An equivalent definition of a rational extension is that each $f \in \operatorname{Hom}_{R}(B, M)$, where $M \supset B \supset C$, can be extended to a unique irreducible partial homomorphism of $M$ into $M$ [3]. It is clear that a rational extension of $C$ is an essential extension.

THEOREM 1 1 . The intersection of all kernels of endomorphisms of $E$ which contain $C$ is the unique (up to isomorphism) maximal rational extension of $C$.

Proof. Let $\mathrm{M}$ be a rational extension of $\mathrm{C}$. The identity mapping of $C$ into $E$ can be extended to an $R$-homomorphism of $M$ into $E$. This homomorphism is an isomorphism since its kernel must have zero intersection with $C$ and $M$ is an essential extension of $C$. Therefore $M$ can be considered as a submodule of $E$. If $f \in \operatorname{Hom}_{R}(E, E)$, where the kernel of $f$ contains $C$, let $K=\{x \in M \mid f(x) \in C\}$. If $f(M) \neq 0$ then $f(M) \cap C \neq 0$ and hence $f(K) \neq 0$. But $f(K)=0$ since $M$ is rational over $C$. Therefore $f(M)=0$, that is $M$ is contained in the kernel of $f$.

Let $\bar{M}$ be the intersection of all kernels of endomorphisms of $E$ which contain $C$. If $\bar{M}$ is not a rational extension of $C$ then there exists a submodule $B, C \subset B \subset \bar{M}$; and $f \in \operatorname{Hom}_{R}(B, \bar{M})$, $f(B) \neq 0$ but $f(C)=0$. Since $E$ is $R$-injective, $f$ can be extended to an endomorphism of $E$ with kernel not containing $M$. This is a contradiction. Thus $\bar{M}$ is the maximal rational extension of C.

DEFINITION 2. A submodule $\mathrm{N}$ of a right $\mathrm{R}$-module $\mathrm{M}$ is called large if $M$ is an essential extension of $N$. A right ideal $A$ of a ring $R$ is called large if $A$ is large considered as a right submodule of $R$.

The proofs of the following two lemmas are either similar to or can be found in the proofs of Theorem 1 and Theorem 2 in [4]. Hence we state them here without proof.

LEMMA 1.

i. If $N_{1}$ and $N_{2}$ are large submodules of $M$ then so is $N_{1} \cap N_{2}$.

${ }^{1}$ It is stated without proof in $[3,2.1]$. 
ii. If $N$ is a large submodule then $T_{m}=\{r \in R \mid m r \in N\}$ is a large right ideal of $R$ for any $m \in M$.

iii. If $A$ is any submodule of $M$ then there exists a submodule $B$ such that $A \cap B=0$ and $A+B$ is large.

An element $\mathrm{x}$ of $\mathrm{C}$ is called a singular element if the annihilator $N_{X}$ of $x$ in $R$ is a large right ideal.

LEMMA 2. The union $J_{R}(C)$ of all singular elements of $C$ is a submodule of $C$.

It is an immediate consequence from the definitions that $J_{R}(M)=0$ if and only if $J_{R}(C)=0$ where $M$ is any essential extension of $C$. If we consider $C$ as an abelian group and $R$ as the ring of integers then $J_{R}(C)$ is the torsion subgroup of $C$.

THEOREM 2. $J_{R}(C)=0$ if and only if each $f \in \operatorname{Hom}_{R}(L, C)$ has a unique irreducible extension in $R$ where $L$ is any large right ideal of $R$.

Proof. Let $f_{1}, f_{2}$ be irreducible extensions of $f \in \operatorname{Hom}_{R}(L, C)$. Suppose $\left(f_{1}-f_{2}\right) x \neq 0, x$ being in the intersection of the domains of $f_{1}$ and $f_{2}$. Then $\left(f_{1}-f_{2}\right) x=c, c \in C, c \neq 0$. Since the intersection of the domains of $f_{1}$ and $f_{2}$ contains $L$ and hence is large, by lemma 1 there exists a large ideal $\mathrm{K}$ of $\mathrm{R}$ such that $x K<$ domain of $f$. If $J_{R}(C)=0$ then $c K \neq 0$. But $c K=\left[\left(f_{1}-f_{2}\right) x\right] K=\left(f_{1}-f_{2}\right)(x K)=0$. Therefore $\left(f_{1}-f_{2}\right) x=0$ for all $x$ in the intersection of the domains of $f_{1}$ and $f_{2}$. By the irreducibility of $f_{1}$ and $f_{2}, f_{1}=f_{2}$ [3].

Conversely, suppose every $f \in \operatorname{Hom}_{R}(L, C)$ has a unique irreducible extension for every large right ideal $L$ of $R$. Given any element $x$ of $C, x$ can be considered as an irreducible homomorphism of $R$ into $C$, therefore, if $x L=0$, where $L$ is a large right ideal of $R$, then $x=0$. Hence $J_{R}(C)=0$.

If we consider the additive group $R^{+}$of $R$ as a right $R$-module, then $J_{R}\left(R^{+}\right)$is an ideal of $R$, called the (right) singular ideal in [4] and [5]. It may be shown that $J_{R}\left(R^{+}\right)=0$ if and only if $R$ is a rational extension of each large right ideal of $R$ (considered as an $\mathrm{R}$-module).

THEOREM 3. If $\mathrm{J}_{\mathrm{R}}(\mathrm{C})=0$ then every essential extension of $\mathrm{C}$ is a rational extension of $\mathrm{C}$. 
Proof. Let $\mathrm{M}$ be an essential extension of $\mathrm{C}$ and $f \in \operatorname{Hom}_{R}(B, M)$, where $M \supset B \supset C$. If $f(C)=0$ but $f(B) \neq 0$, then there exists some $b \in B$ such that $f(b) \in C$ and $f(b) \neq 0$. The set $T_{b}=\{r \in R \mid b r \in C\}$ is a large right ideal of $R$ such that $f(b) T_{b}=f\left(b T_{b}\right)=0$. This contradicts the as sumption that $J_{R}(C)=0$, and therefore $f=0$ whenever $f(C)=0$. This proves the theorem.

LEMMA 3. If $C$ is a rational extension of $C_{1}$ and $C_{2}$, then: i. $\quad C$ is a rational extension of $\mathrm{C}_{1} \cap \mathrm{C}_{2}$. ii. If $f \in \operatorname{Hom}_{R}\left(C_{2}, C\right)$ and $D=\left\{x \in C_{2} \mid f(x) \in C_{1}\right\}$, then $C$ is a rational extension of $D$.

\section{Proof. See Propositions 1.1 and 1.2 in [3].}

Let $M$ be the unique maximal rational extension of $C$ and let $S$ be the set of all irreducible fractional homomorphisms of $M$ into $M$ [3]. Thus, $f \in S$ if $M$ is rational over the domain of $f$.

THEOREM 4. S is a ring with an identity element and it contains a subring isomorphic with the ring $\operatorname{Hom}_{R}(C, C)$.

Proof. According to [3; Section 4], $S=\operatorname{Hom}_{R}(M, M)$ and therefore $S$ is a ring with an identity element. Since each $f \in \operatorname{Hom}_{R}(C, C)$ has a unique extension in $S, \operatorname{Hom}_{R}(C, C)$ is isomorphic to a subring of $\mathrm{S}$.

COROLLARY. If $J_{R}(C)=0$ then $S=\operatorname{Hom}_{R}(E, E)$ where $E$ is the maximal essential extension of $C$.

Proof. If $J_{R}(C)=0$ then $M=E$ 。

THEOREM 5. If $J_{R}(C)=0$ then $S$ is a regular ring [7] and self-injective (considered as a right $\mathrm{S}$-module).

Proof. The zero singular submodule of $\mathrm{C}$ implies $J_{R}(M)=0$. Therefore $M$ is a rational extension of any large submodule of $M$. So if $x \in S$, let $N$ be a submodule of $M$ sinch that $N \cap N_{X}=0$ and $N+N_{X}$ is large where $N_{X}=\{m \in M \mid x m=0\}$. Now $\mathrm{x}$ is an isomorphism on $\mathrm{N}$, so there exists a mapping y such that $y(x a)=a$ for all $a$ in $N$. Thus $y$ is an R-homomorphism of $x N$ into $M$. By the injectivity of $M$, $y$ can be extended to an element $\bar{y}$ in $S$. Since $\bar{y}(x t)=0$ for all $t$ in $N_{x},(x \bar{y} x-x) w=0$ for all $w$ in $N+N_{X}$. Since $N+N_{X}$ is large, therefore $x \bar{y} x=x$. Thus $S$ is a regular ring. 
To prove $S$ is self-injective, we must show that for each right ideal $A$ of $S$ and $\varphi \in \operatorname{Hom}_{S}(A, S)$ there exists $z \in S$ such that $\varphi \mathrm{a}=$ za for all a in $\mathrm{A}[2$, Theorem 3.2]. If $A M=\left\{\sum_{i} a_{i} x_{i} \mid a_{i} \in A, x_{i} \in M\right\}$, define the mapping $\eta$ of $A M$ into $M$ as follows:

$$
\eta\left(\Sigma_{i} a_{i} x_{i}\right)=\Sigma_{i}\left(\varphi a_{i}\right) x_{i}
$$

This mapping is well defined, for if $\Sigma_{i} a_{i} x_{i}=0$ choose e as the idempotent generator of the ideal $a_{1} S+a_{2} S+\ldots \ldots+a_{n} S$. This can be done since $S$ is a regular ring $[7$, lemma 15]. Then $e a_{i}=a_{i}, e \in A$ and $\sum_{i}\left(\varphi a_{i}\right) x_{i}=\sum_{i}(\varphi e) a_{i} x_{i}=0$. Clearly $\eta$ is an $R$-homomorphism of $A M$ into $M$. Hence $\eta$ can be extended to an element $\mathrm{z}$ of $\mathrm{S}$ and $(\varphi(\mathrm{a})) \mathrm{x}=\eta(\mathrm{ax})=z(\mathrm{ax})=(\mathrm{za}) \mathrm{x}$, for all $\mathrm{x}$ in $\mathrm{M}$. Thus $\varphi \mathrm{a}=\mathrm{za}$ for all $\mathrm{a}$ in $\mathrm{A}$. This completes the proof.

Suppose $\mathrm{C}$ is a ring and $\mathrm{C}$ is a left $\mathrm{C}$ and right $\mathrm{R}$ bimodule. Then $C$ can be considered as a subring of $S$ if for all $c \in C$, $\mathrm{cC}=0$ implies $c=0$ ( $\mathrm{C}$ is left-faithful).

THEOREM 6. If $\mathrm{C}$ is a left-faithful ring and a left $\mathrm{C}$ right $R$ bimodule then $S$ is a rational extension of $C$ as a right $C$-module.

Proof. Let $f \in \operatorname{Hom}_{C}(B, S)$ where $C \subset B \subset S$ and $f(C)=0$.

Suppose $f \neq 0$, then there exists $b \in B$ such that $f(b) \neq 0$. $M$ is rational over the intersection of $b-1$ and $C$ and hence is rational over $T$ where $T=\left\{x\right.$ is in the intersection of $b^{-1} C$ anc $C \mid b x \in C\}$. Hence $f(b) T \neq 0$. However $f(b) T=f(b T)=0$. Therefore $f=0$ and $S$ is a rational extension of $C$ as a right C-module.

A ring $Q$ is called a right ring of quotients of a ring $C$ if $Q \supset C$ and $Q$ is rational over $C$ as a right $C$-module [3]. This definition coincides with $R$. E. Johnson's definition [4] in the case when $J_{C}(C)=0$.

LEMMA 4. Suppose $C$ is a ring and $C$ is rational over $R$ as a right $R$-module and $J_{R}(C)=0$, then any rational extension of $C$ as $C$-module is a rational extension of $C$ as $R$-module.

Proof. Let $\mathrm{K}$ be a rational extension of $\mathrm{C}$ as a right $\mathrm{C}$-module. $\mathrm{K}$ is an $\mathrm{R}$-extension of $\mathrm{C}$. Since $\mathrm{J}_{\mathrm{R}}(\mathrm{C})=0$, we only have to show that $\mathrm{K}$ is an $\mathrm{R}$-essential extension of $\mathrm{C}$. For any $k \in K, k \neq 0$, there exists $c, c^{\prime}$ in $C, c^{\prime} \neq 0$, and $k c=c^{\prime}$. 
Consider the large right ideal $T$ of $R$, where $T=\{r \in R \mid c r \in R\}$, then $c^{\prime} T \neq 0$. In other words $k R \cap C \neq 0$, thus $K$ is an essential extension of $\mathrm{C}$ as a right $\mathrm{R}$-module.

THEOREM 7. If $\mathrm{C}$ is a ring and $\mathrm{C}$ is rational over $\mathrm{R}$ as a right $R$-module and $J_{R}(C)=0$, then $C$ can be imbedded into a right self-injective ring $S$ where $S$ is a regular ring and the right maximal ring of quotients of $C$.

Proof. Any ring of quotients of $\mathrm{C}$ can be considered as a rational extension of $C$ as a right $R$-module by the above lemma. We already showed that $S=\operatorname{Hom}_{R}(E, E)$ is a right ring of quotients of $C$. The rest of the proof follow from Theorem 5 .

Let $R$ be a ring with an identity element. Consider the additive group $R^{+}$of $R$ as a right $R$-module.

LEMMA 5. If $M$ is an essential extension of $R^{+}$(as a right (ile) then $M$ is rational over $R^{+}$if and only if $R^{+}$is rational over $\mathrm{Tm}$ for all $\mathrm{m}$ in $\mathrm{M}$, where $\mathrm{T}_{\mathrm{m}}=\left\{\mathrm{r} \in \mathrm{R} / \mathrm{mr} \in \mathrm{R}^{+}\right\}$.

Proof. Suppose $R^{+}$is rational over $T_{m}$ for all $m$ in $M$. Let $f \in \operatorname{Hom}_{R}(B, M), M \supset B \supset R^{+}$and $f\left(R^{+}\right)=0$. If $f \neq 0$ then $f(B) \cap R^{+} \neq 0$. Hence there exists $b \in B, r \in R$, such that $r \neq 0$ and $f(b)=r$. Now $f(b) T_{b}=r T_{b} \neq 0$, for $R$ is rational over $T_{b}$. But $b T_{b}<R^{+}$and $f(b) T_{b}=f\left(b T_{b}\right)=0$. Therefore $f=0$ and $M$ is rational over $R^{+}$.

Conversely, let $M$ be a rational extension of $R^{+}$and let $f \in \operatorname{Hom}_{R}\left(B, R^{+}\right)$where $R^{+} \supset B \supset T_{m}$ for any $m$ in $M$ and $f\left(T_{m}\right)=0$. Consider the submodule $R^{+}+m B$. Define $\varphi(r+m b)=f(b)$. If $r+m b=0, b \in T_{m}$ and hence $f(b)=0$. Thus the mapping $\varphi$ is well defined. But $\varphi\left(R^{+}\right)=0$, therefore $\varphi=0$ and $f=0$. Thus $R^{+}$is rational over $T_{m}$ for all $\mathrm{m}$ in $\mathrm{M}$.

Let $L$ be the left maximal ring of quotients of $R$ and $Q$ be the right maximal ring of quotients of $R$.

Consider $L$ as a right $R$-module and let $T$ be the set of all elements $x$ in $L$ for which there exists a right ideal $A$ of $R$. such that $x A \subset R$ and $R$ is rational over $A$.

THEOREM 8. $T$ is a subring of $L$ and $T$ is the unique (up to isomorphism over $R$ ) left and right maximal ring of quotients of $R$. 
Proof. If $x$ and $y$ are in $T$, let $A_{x}$ and $A_{y}$ be the right ideals of $R$ such that $x A_{x} \subset R, y A_{y} \subset R$ and $R$ is rational over $A_{x}$ and $A_{y}$. Since $R$ is rational over $A_{x} \cap A_{y}$ and $(x-y)\left(A_{x} \cap A_{y}\right) \subset R, x-y$ is in $T$. Also $R$ is rational over $D$, where $D=\left\{r \in A_{y} \mid\right.$ yr $\left.\in A_{x}\right\}$ and $x y D \subset R$. Therefore $x y$ is in $T$. This proves $T$ is a subring of $L$. It is clear that $T$ contains the identity element of $L$. If $x \in T, A_{x} \subset T_{x}$. Thus $R$ is rational over $T_{X}$ for all $x$ in $T$. By lemma $5, T$ is rational over $R$ and hence a right ring of quotients of $R$.

To show $T$ is the left and right maximal ring of quotients of $R$, let $K$ be a left and right ring of quotients of $R$. Then $\mathrm{K} \subset \mathrm{L}$ and also $\mathrm{K} \subset \mathrm{T}$, since for every $\mathrm{x}$ of $\mathrm{K}$ there exists a right ideal $T_{x}$ of $R$ such that $x T_{X}<R$ and $R$ is rational over $T_{X}$.

If $R$ is a commutative ring, any left $R$-module can be considered as a right $R$-module. In this case, the left maximal ring of quotients of $R$ coincides with the right maximal ring of quotients of $R$.

\section{REFERENCES}

1. B. Eckmann and A. Schopf, Über injektive Moduln, Archiv der Math. 4 (1953), 75-78.

2. H. Cartan and S Eilenberg, Homological algebra, (Princeton, 1956).

3. G.D. Findlay and J. Lambek, A generalized ring of quotients I, II, Can. Math. Bull. 1 (1958), 77-85, $155-167$.

4. R.E. Johnson, The extended centralizer of a ring over a module, Proc. Amer. Math. Soc. 2 (1951), 891895.

5. R. E. Johnson, Structure theory of faithful rings II, Trans. Amer. Math. Soc. 84 (1957), 523-542.

6. Y. Utumi, On quotient rings, Osaka Math. J. 8 (1956), 1-18.

7. J. von Neumann, On regular rings, Proc. Nat. Acad. Sci. U.S.A. 22(1936), 707, ?13.

Oberlin College, U.S.A.

Smith Coliege, U.S.A. 